Revue des patrimoines

$24 \mid 2014$

Architecture et urbanisme de villégiature : un état de la recherche

\title{
Architecture et urbanisme de villégiature : un état de la recherche \\ Éditorial
}

Claude Laroche, Bernard Toulier, Éric Cron et Franck Delorme

\section{OpenEdition \\ Journals}

Édition électronique

URL : http://journals.openedition.org/insitu/11099

DOI : 10.4000/insitu. 11099

ISSN : 1630-7305

Éditeur

Ministère de la Culture

\section{Référence électronique}

Claude Laroche, Bernard Toulier, Éric Cron et Franck Delorme, «Architecture et urbanisme de

villégiature : un état de la recherche », In Situ [En ligne], 24 | 2014, mis en ligne le 17 juillet 2014, consulté le 22 septembre 2020. URL : http://journals.openedition.org/insitu/11099 ; DOI : https:// doi.org/10.4000/insitu.11099

Ce document a été généré automatiquement le 22 septembre 2020

\section{(c) $(1)$}

In Situ Revues des patrimoines est mis à disposition selon les termes de la licence Creative Commons Attribution - Pas d'Utilisation Commerciale - Pas de Modification 4.0 International. 


\section{Architecture et urbanisme de villégiature : un état de la recherche} Éditorial

Claude Laroche, Bernard Toulier, Éric Cron et Franck Delorme

1 Dix ans après le précédent numéro d'In Situ consacré à la villégiature, les points de vue sur la question ne semblent pas fondamentalement différents, même si, comme le montre Claude Mignot, les perspectives se sont sensiblement élargies. Si changement il y a, il serait peut-être plus patrimonial que scientifique, davantage lié à la perception des «objets» de la villégiature qu'aux préoccupations des chercheurs. Il faut bien reconnaître que désormais, même si le combat patrimonial est toujours d'actualité, ces objets sont assez souvent en phase avec le goût d'un public qui se laisse volontiers porter par ses aspects aimables. Les «villes d'art et d'histoire » concernées - et rares sont celles, serions-nous tentés de dire, qui ne sont pas touchées peu ou prou par le phénomène - font souvent de la question de la villégiature un axe privilégié de leur politique de valorisation patrimoniale. On est loin désormais du temps où le $\mathrm{XIX}^{\mathrm{e}}$ siècle était méprisé, du temps où le $\mathrm{xx}^{\mathrm{e}}$ siècle ne pouvait prétendre au statut patrimonial, où il était impensable d'examiner sereinement l'architecture des Trente glorieuses. Pour autant, ce relatif engouement pour le sujet ne va pas sans son lot d'idées reçues et de clichés ; par conséquent, ce succès ne peut faire oublier que la recherche fondamentale se doit d'enrichir encore et toujours la connaissance sur un phénomène capital pour la compréhension fine des sociétés des trois derniers siècles.

2 Tentant de faire un point, toujours provisoire et éminemment perfectible, ce numéro s'est nourri initialement de deux rencontres, deux moments de bilans et d'échanges entre les chercheurs. À Biarritz, du 12 au 14 octobre 2010, la direction générale des Patrimoines du ministère de la Culture et la région Aquitaine unirent leurs forces pour proposer une formation intitulée : «Villégiature : l'architecture à l'épreuve du site ». Elle se situait explicitement dans le prolongement des rencontres ayant eu lieu selon un rythme à peu près biennal depuis le début des années 1990, comme le rappelle opportunément ici-même Claude Mignot. Tout en jouant son rôle de bilan et bien sûr, de formation destinée aux professionnels du patrimoine, elle illustrait deux axes 
principaux, chronologique pour l'un, thématique pour l'autre. Prenant acte de ce que les périodes récentes de la villégiature étaient désormais devenues objets d'étude, elle faisait toute sa part à la deuxième moitié $d u x^{e}$ siècle - qui n'était toutefois pas absente des premières rencontres (La Grande-Motte, présentée en 1993, à Biarritz déjà). Il paraissait intéressant, entre autres, de voir comment les premières stations, celles qui s'étaient conformées à une image "pittoresque ", arborée, une image centrée sur le type de la villa isolée en milieu de parcelle au sein d'un urbanisme assez lâche, comment ces stations avaient abordé une période, les Trente glorieuses, qui se défiait du pittoresque, qui réduisait la place de la végétation ou qui se densifiait pour répondre aux nouveaux besoins. Des stations qui furent donc alors confrontées à un vrai dilemme : comment conforter, amplifier une fonction de villégiature, s'exerçant dans le domaine du loisir, une fonction forte qui faisait d'elles des villes spéciales, alors que dans le même temps, leur importance en faisait aussi de vraies villes, avec des besoins en termes d'administration et d'équipement de villes normales. Ce sont ces villes prises entre deux destins - par exemple Arcachon - qu'il était intéressant d'observer à ce moment crucial de leur histoire.

3 Le deuxième axe était donc thématique. Une composante de la formation de l'urbanisme et de l'architecture de la villégiature nous paraissait avoir assez peu été examinée en soi : le rapport au lieu, au site. Certes, la question, assez rebattue, du génie du lieu a souvent été au point de départ des études sur la villégiature ${ }^{1}$, mais la question des prises de position architecturales et urbaines face au site, attitudes adoptées par les concepteurs des édifices ou des stations, nous paraissait encore un champ relativement peu exploré. Si cette question importe pour tout le domaine architectural et urbain, elle prend évidemment une dimension toute particulière s'agissant des lieux de loisir. La villégiature, c'est d'abord et avant tout un site, lequel doit être ou majestueux, ou prenant, ou aimable. Or, le site de villégiature possédera ces qualités... tant qu'il restera vierge ! L'étude de l'équilibre indispensable entre l'occupation, la mise à profit d'un site d'exception et sa relative préservation - sous peine de tuer la poule aux œufs d'or -, voire sa sublimation, était l'un des axes d'études recherchés.

4 Mais ce qui peut paraître une contrainte majeure, dès lors que l'on raisonne en termes de préservation, peut aussi se révéler source d'inspiration. La morphologie du site, ses caractéristiques de tous ordres peuvent le faire passer du statut de paysage fragile, à préserver, à celui de moteur, d'élément déclencheur de la conception. Il peut alors devenir source première du « parti » (une notion qui a eu son heure de gloire avant son remplacement par celle de «concept »...) architectural ou urbain, que les créateurs aient tenté une sorte de mimétisme ou, au contraire, joué du contraste, de la rupture. C'est là que se situe l'analyse des rapports des bâtiments aux points de vue, aux éléments climatiques (le balcon de cure ou de villégiature, tel que l'analyse ici PierreLouis Laget) et aux autres composantes fortes du site ; là que prend place l'examen de l'adaptation des types courants en fonction des différentes vues. La recherche, on le voit, a encore un vaste champ d'action devant elle. Notamment en matière d'analyse des formes architecturales: on pense entre autres à ce qu'évoquait Claude Mignot au moment de conclure les journées de Biarritz, quand il remarquait la permanence d'une sorte de «culture de la toiture " souvent légitimée par la référence au lieu et qui concerne autant la villa anglo-normande du $\mathrm{XIX}^{\mathrm{e}}$ siècle et du début $\mathrm{du} \mathrm{xx}^{\mathrm{e}}$ que les architectures d'Avoriaz... 
Les deux axes pouvaient s'entremêler : le second $\mathrm{xx}^{\mathrm{e}}$ siècle est l'époque où apparaissent des notions comme celle "d'intégration » au site, notion très ambiguë, qui pouvait sous-entendre toutes sortes de prises de position par rapport à l'environnement : des démarches mimétiques - si tant est qu'elles puissent exister - ou des recherches "d'harmonie » entre le bâti et le paysage - terme là encore très ambivalent; une époque où fleurissaient encore des attitudes architecturales et urbaines plus « brutalistes » qui ne sont évidemment pas indifférentes à l'environnement puisqu'elles réagissaient expressément à celui-ci (l'hôtel Le Flaine de Marcel Breuer, le VVF d'Anglet de Jean-Raphaël Hébrard et André Grésy ou le Victoria Surf de Biarritz de Jean-Claude Lesgourgues (fig. 1, 2, 3).

Figure 1

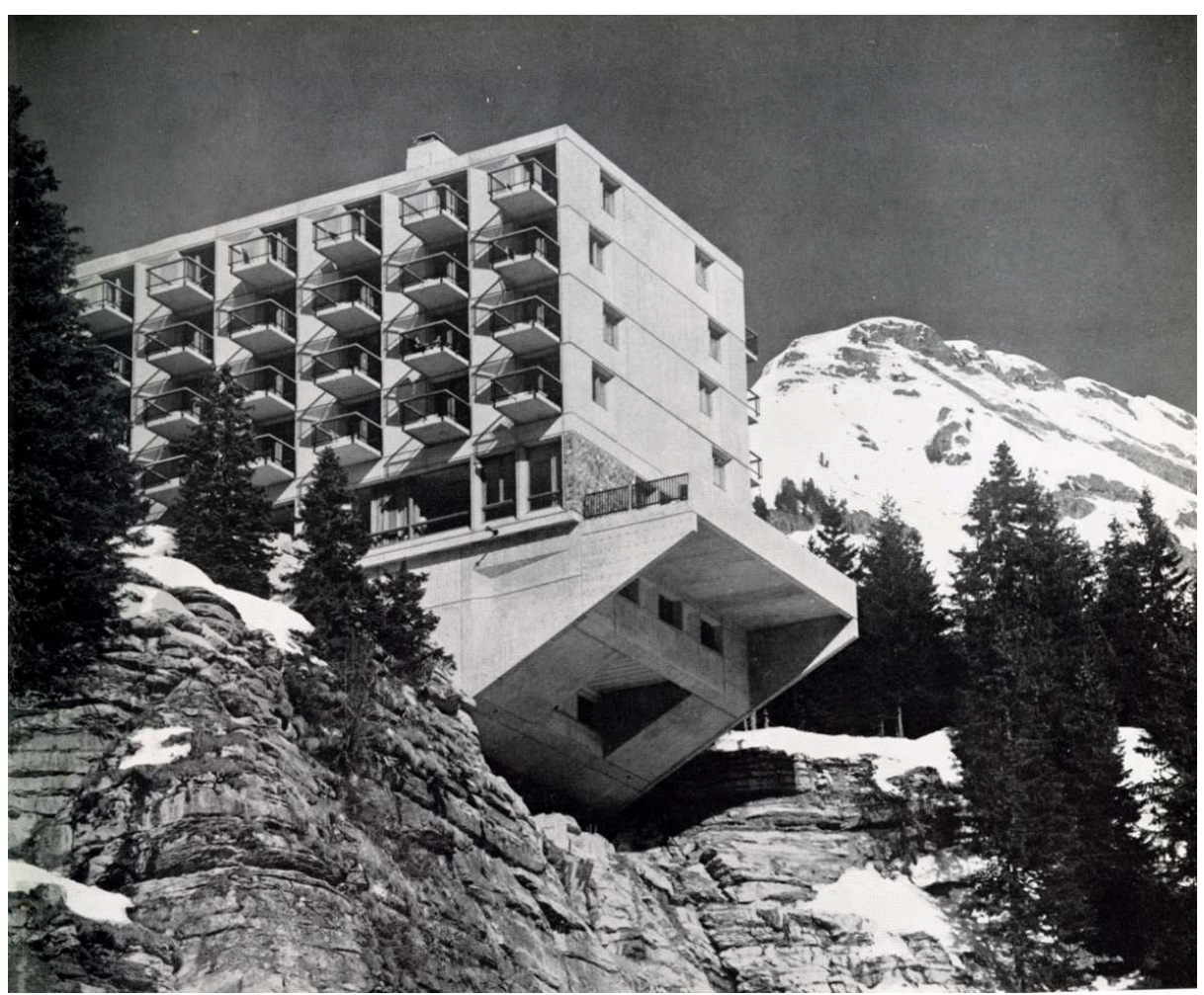

Arâches-la-Frasse (Haute-Savoie) : hôtel Le Flaine, par Marcel Breuer, 1966-1968. Extrait de Techniques et Architecture, $1969, n^{\circ} 4$. 
Figure 2

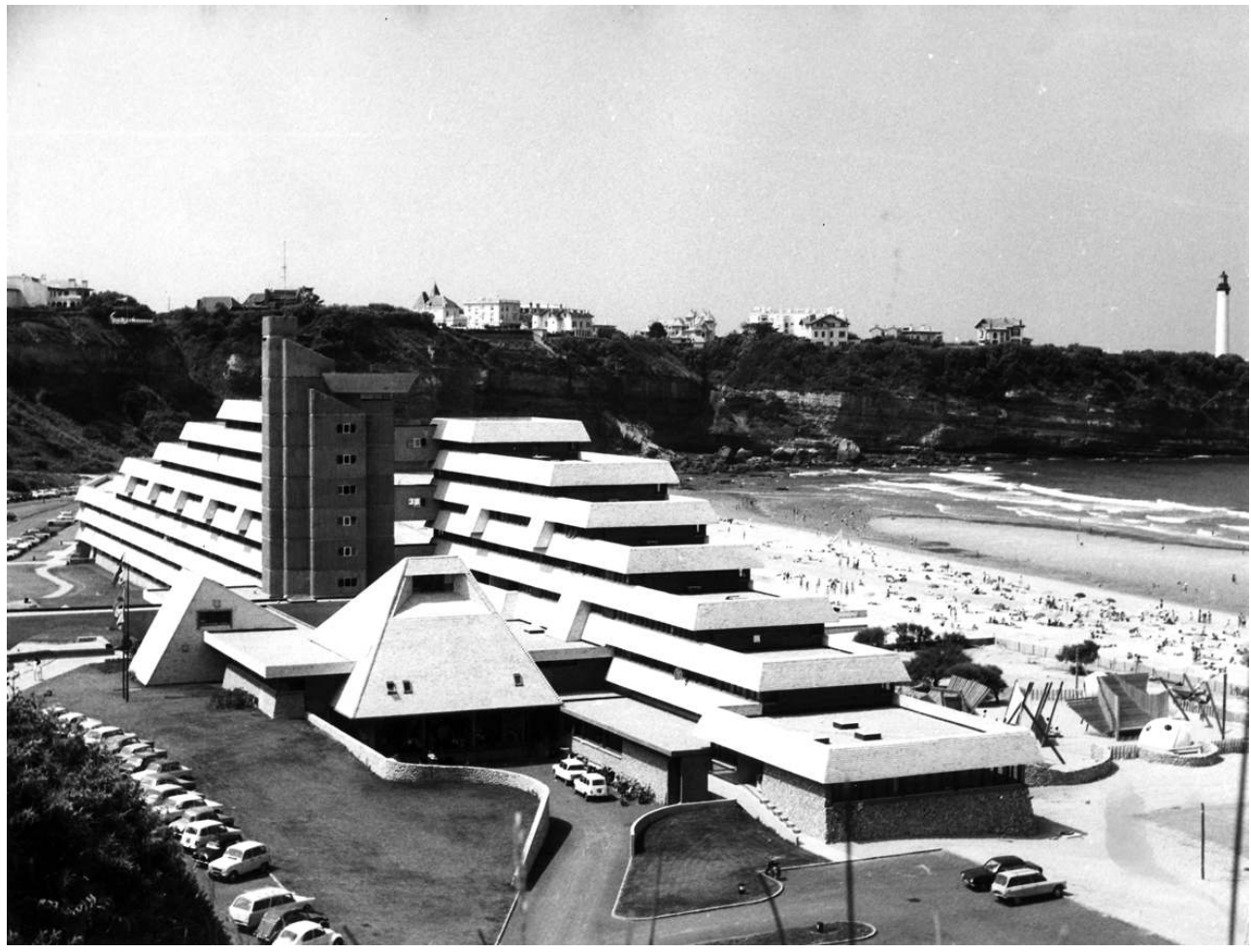

Anglet (Pyrénées-Atlantiques) : Village vacances famille (VVF), par Aquitaine architectes associés (dont Jean-Raphaël Hébrard et André Grésy), 1969, vue d'ensemble depuis la falaise. N.d., Siaf/Capa/ Archives d'architecture du xx siècle, fonds DAU, 133 Ifa 7/1.

\section{Figure 3}

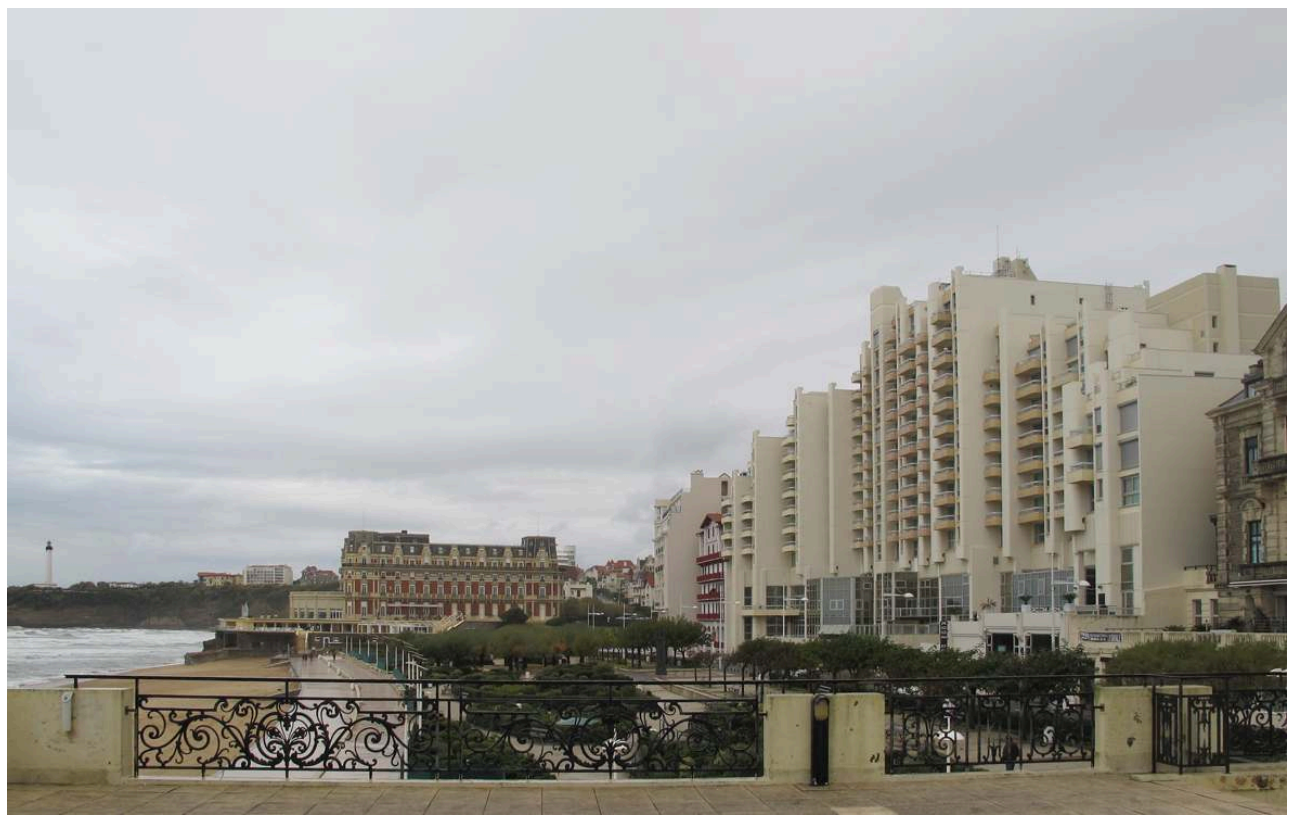

Biarritz (Pyrénées-Atlantiques) : à droite, immeuble Victoria Surf, par Jean-Claude Lesgourgues, 1974-1976, ensemble depuis le casino municipal ; à l'arrière-plan, l'hôtel du Palais, construit à l'image et sur l'emplacement de la villa Eugénie par Édouard-Jean Niermans.

Phot. Laroche, Claude, 2012. (c) Claude Laroche. 
6 La deuxième rencontre eut lieu quelques mois plus tard, le 11 juin 2011, à Paris. Elle fut organisée au palais de Chaillot par la Cité de l'architecture et du patrimoine sous la forme d'une journée d'étude intitulée « De la villégiature aux lieux de loisirs ». Elle se proposait d'étudier ces « espaces hors du quotidien » créés continuellement depuis le $\mathrm{XIX}^{\mathrm{e}}$ siècle, ces lieux d'expérimentation allant jusqu'à l'utopie. Sous la forme d'exposés et d'une table ronde, elle explorait autant l'histoire et les enjeux patrimoniaux que les questions contemporaines liées à la réhabilitation, à l'aménagement, aux mutations et à la création. Elle examinait le patrimoine de la villégiature comme enjeu identitaire des politiques territoriales et comme facteur de développement économique. Elle suggérait enfin quelques réflexions autour de la mondialisation des loisirs, de la multiplication des échanges et de la conformité des nouveaux objets de la villégiature à quelques modèles en nombre limité.

7 Les différentes contributions à ces deux rencontres ainsi que divers articles indépendants ont permis de bâtir un numéro qui fait la part belle à tous ces thèmes ${ }^{2}$. C'est ainsi qu'on trouvera un axe de réflexion portant sur les nouveaux regards que peuvent susciter les sites pionniers dans le domaine de la villégiature. Que ce soient des retours sur certains débuts (la Côte d'Azur, Aix-les-Bains, Enghien) ou un panorama permettant d'embrasser un thème sur la longue durée (les stations thermales) ou sur un territoire important (l'île-de-France). Que ce soit aussi la mise en évidence de la permanence des données initiales (Arcachon, où le primat du climatique perdure, même paré de nouveaux habits). Que ce soit enfin l'observation de quelques "isolats » au sein d'entités de villégiature par ailleurs bien établies, des isolats liés à de fortes personnalités: Antoine d'Abbadie à Hendaye, qui dans son incessante quête de l'ailleurs, en oublierait presque le site grandiose qui accueille son château; Kitty Abry à La Valmasque, inscrite dans une démarche spirituelle comparable et tout aussi indifférente à l'environnement balnéaire, ici de la Côte d'Azur.

8 Le sujet central de la rencontre de Biarritz, "la villégiature à l'épreuve du site ", se devait d'occuper une place importante: il fournit en effet la matière du deuxième thème de ce numéro. Les idées suggérées plus haut y sont en partie traitées grâce à des présentations fécondes, qui sont autant de guides pour les recherches à venir. Ici, la forme de l'interview, une " première " pour In Situ, a paru la mieux à même de traduire les desseins de Jacques Labro, le principal concepteur d'Avoriaz.

9 Le troisième thème, quant à lui, réunit des interventions de Biarritz (les aménagements des côtes languedocienne et aquitaine - là encore, s'agissant de cette dernière, sous la forme d'une interview) et de Paris pour voyager du réel à l'utopie. Tout domaine de recherche est tributaire de ses sources spécifiques: c'est le dernier thème de ce numéro, auquel on a rattaché la question des collections de la Cité de l'architecture et $\mathrm{du}$ patrimoine, un point sur la question des guides de voyage ainsi qu'un bilan bibliographique des services d'Inventaire.

La région Aquitaine, indépendamment des exemples de son territoire exposés ici (Arcachon, Hendaye, l'aménagement de la côte aquitaine), s'est tout particulièrement impliquée dans ce numéro. Elle se sentait une forte légitimité pour cela, à la fois par l'histoire du service régional de l'Inventaire que par son programme de recherche, présent et à venir. Après avoir consacré, dès sa création en 1967, ses premières recherches à l'étude du patrimoine en milieu rural, le service aquitain entreprit en effet en 1985 une étude pionnière sur la station balnéaire d'Hossegor, annonçant ainsi ce qui allait devenir l'un de ses thèmes de recherche de prédilection. Les objectifs de cet 
inventaire, qui resta topographique malgré l'importance de la villégiature sur ce territoire, furent de poser les bases d'une réflexion sur l'histoire des stations balnéaires en Aquitaine et sur le régionalisme architectural, en l'occurrence néo-basco-landais. À la suite de cette opération, le service entreprit dans les années 1990 une étude ambitieuse sur l'architecture de la villégiature de la côte basque, des années 1850 à 1950. Elle permit non seulement de dresser un portrait éloquent de l'espace et de l'architecture de la villégiature, mais aussi d'asseoir sur un corpus significatif l'étude de phénomènes urbains ou architecturaux importants qui dépassent, par leurs enjeux, le strict cadre local (formation de la ville balnéaire, mise en place des équipements aux $\mathrm{XIX}^{\mathrm{e}}$ et $\mathrm{XX}^{\mathrm{e}}$ siècles, mise au point et diffusion d'une architecture régionaliste, etc.). Près de cinq mille édifices ont été ainsi recensés dans ce cadre.

11 Fort de cette spécialité scientifique acquise au fil des années, le service régional du patrimoine et de l'Inventaire a décidé de poursuivre dans cette voie (accompagnement d'une thèse sur la Mission interministérielle d'aménagement de la côte aquitaine, inventaire topographique en cours de la commune d'Arcachon et des communes riveraines de l'estuaire de la Gironde, etc.). Afin d'appréhender le phénomène de la villégiature dans toutes ses composantes, un inventaire topographique de la ville de Pau a été également lancé, nourrissant le portrait d'une région « terre de villégiature ».

Mais à vrai dire, "terres de villégiature ", toutes les régions françaises le sont à un degré ou à un autre. C'est bien là, lié à l'élargissement du champ d'étude, le principal enseignement de ces quelque trente ans de recherche: comment un thème qui, se rapportant aux loisirs, à l'otium, pouvait paraître à première vue quelque peu futile, s'est révélé être une clef d'entrée privilégiée à qui veut connaître les fondements d'une société, l'esprit d'une époque. Dans une activité librement choisie, abritée par une architecture distinctive face à des paysages d'exception: c'est peut-être ici que l'on pourrait le mieux appréhender le comportement humain à un moment donné.

\section{NOTES}

1. - LAROCHE, Claude. "Le Génie du lieu et l'Esprit du temps". Dans Architecture et identité régionale: Hossegor, 1923-1939. Bordeaux: Inventaire général des monuments et des richesses artistiques de la France, APIA, Le Festin, 1993 (coll. Cahiers du patrimoine, n 32), p. 19.

2. - Ce numéro doit beaucoup à l'important travail de relecture effectué par Catherine Gros, véritable travail éditorial, autant de forme que de fond. 


\section{AUTEURS}

\section{CLAUDE LAROCHE}

chercheur, Ingénieur territorial, Conseil régional d'Aquitaine, Direction de la Culture et du Patrimoine, Service régional du Patrimoine et de l'Inventaire claude.laroche@aquitaine.fr

\section{BERNARD TOULIER}

conservateur général honoraire du patrimoine btoulier@gmail.com

\section{ÉRIC CRON}

Chef du service régional du Patrimoine et de l'Inventaire, Direction de la Culture et $\mathrm{du}$ Patrimoine, Région Aquitaine eric.cron@aquitaine.fr

\section{FRANCK DELORME}

attaché de conservation, Cité de l'architecture et du patrimoine, centre d'archives d'architecture du xx siècle/Ifa fdelorme@citechaillot.fr 